Running head: SHARED SIGNALS

\title{
IDENTIFYING PERSONALITY FROM THE STATIC, NON-EXPRESSIVE FACE IN HUMANS AND CHIMPANZEES: EVIDENCE OF A SHARED SYSTEM FOR SIGNALLING PERSONALITY
}

\author{
ROBIN S. S. KRAMER ${ }^{a}$, JAMES E. KING ${ }^{b}$, ROBERT WARD ${ }^{\mathrm{a}, *}$
}

${ }^{\text {a } B a n g o r ~ U n i v e r s i t y, ~ B a n g o r, ~ G w y n e d d, ~ U K ~}$

${ }^{\mathrm{b}}$ University of Arizona, Tucson, Arizona, USA

* Corresponding author.

Postal address: Robert Ward, Wolfson Centre for Clinical and Cognitive Neuroscience, School of Psychology, Bangor University, LL57 2AS, UK.

E-mail address: r.ward@bangor.ac.uk (R. Ward).

Word count: 4212 
A shared signal system 2

\begin{abstract}
Many aspects of personality are honestly signalled on the human face, as shown by accurate identification of personality traits from static images of unknown faces with neutral expressions. Here we examine the evolutionary history of this signal system. In four studies, we found that untrained human observers reliably discriminated characteristics related to extraversion solely from non-expressive facial images of chimpanzees (Pan troglodytes). In chimpanzees, as in humans, there is therefore information in the static, non-expressive face that signals aspects of an individual's personality. We suggest this performance is best explained by shared personality structure and signalling in the two species.
\end{abstract}


A shared signal system 3

\section{Introduction}

Personality traits describe the stable, context-general behavioural biases of an individual organism. Factor-analytic approaches have identified a small number of human personality traits, three-factor (Eysenck \& Eysenck, 1985) and five-factor models (Goldberg, 1993) being the most used. Human personality as defined by these models has cultural but also biological bases. Behaviour genetics studies estimate heritability coefficients for individual traits around $.40-.60$ (Bouchard \& Loehlin, 2001), and human models show cross-cultural and even crossspecies generalisation of personality factors (Gosling \& John, 1999). In particular, humans and chimpanzees demonstrate similar, although not identical, factor structures. The most important distinction is that the factor-analytic approach identifies an additional (King \& Figueredo, 1997), highly heritable (Weiss, King, \& Figueredo, 2000) dominance-related factor present in chimpanzees but not humans.

Many socially-relevant traits can be accurately identified in humans solely from visible cues in the static, non-expressive face, including personality (Kramer \& Ward, 2010; Little \& Perrett, 2007), sociosexuality (Boothroyd, Jones, Burt, DeBruine \& Perrett, 2008), trustworthiness (Stirrat \& Perrett, 2010), and aggression (Carré, McCormick \& Mondloch, 2009). Interpreting these results within animal signalling theory suggests a close association between the facial morphology and behaviour of the signal "sender", and the cognitive processes for understanding the signal in the "receiver" (Maynard-Smith \& Harper, 2003). Here we examine the evolutionary history of this signal system. We reasoned that if the face were part of an evolved signal system, then humans and chimpanzees might share aspects of this system. If so, some facial morphology signals should be expressed and understood between species. 
The possibility of a shared signal system is plausible in part due to evidence from comparative studies showing similarities in face processing for the two species, including homologous specialised brain regions (Parr, Hecht, Barks, Preuss \& Votaw, 2009), crossspecies identification of relatedness (Alvergne et al., 2009), sensitivity to facial configurations (Parr, Heintz \& Akamagwuna, 2006), and homologies in expression (Parr, Waller, Vick \& Bard, 2007). However, a shared signal system of personality would require a variety of other physical and psychological homologies, including behavioural biases as reflected by aspects of personality structure, facial morphology, and the cognitive means for correctly interpreting and using these signals from the face.

To test the possibility of a shared signal system, we measured the ability of humans to understand signals from the static chimpanzee face, related to extraversion. Our focus on extraversion was motivated by previous findings. First, in the human face, the signal for extraversion is strong, and apparent in both individual (Penton-Voak, Pound, Little \& Perrett, 2006) and composite faces (Kramer \& Ward, 2010). Second, personality characteristics related to human extraversion, such as individual differences in dominance, sociability, and activity levels, are widespread in non-human animals (Gosling \& John, 1999), including other primates (e.g. chimpanzees; King \& Figueredo, 1997), other mammals (e.g. hyenas; Gosling, 1998), fish (e.g. guppies; Budaev, 1997), and even invertebrates (e.g. octopuses; Mather \& Anderson, 1993). Finally, the characteristic of 'dominant' is encompassed by the trait of Extraversion in the human taxonomy (Goldberg, 1990), and is a particularly robust measure in chimpanzees, demonstrating the single highest factor weighting of any characteristic in the chimpanzee personality model (King \& Figueredo, 1997), and both reliability (Freeman \& Gosling, 2010), and external validity in predicting individual behaviour (Pederson, King \& 
Landau, 2005). We carried out a series of four studies in order to investigate accuracy in identifying characteristics relating to extraversion from static chimpanzee faces.

\section{Study 1: accurate personality identification from the chimpanzee face}

The first study determined whether people could accurately perceive characteristics relating to extraversion and other personality traits from chimpanzee facial photographs using a forced-choice methodology.

\subsection{Method}

\subsubsection{Participants}

Forty-three students from Bangor University (age 19-50 years, 26 female) took part in exchange for course credit.

\subsubsection{Stimuli}

We obtained an initial set of 37 photographs of chimpanzees, each with previously collected personality information as described by King and Figueredo (1997). The photographs were mostly full body images and showed the chimpanzees in a natural setting. Chimpanzees were either looking straight at the camera or in three-quarter view, and the images were mirrored as necessary so that all angled to their left. All images were cropped to show only the head, with a small amount of neck/body and background remaining (Fig. 1A). We selected the images without valenced facial expressions, e.g. without teeth visible or 
strong shadowing over the eyes. Images were approximately 300 x 300 pixels in size, or about an $8.5 \mathrm{~cm}$ square on the screen.

We selected 15 of the 43 characteristics assessed in the King and Figueredo (1997) database. These characteristics were chosen in order to represent the six chimpanzee personality factors (King \& Figueredo, 1997): the two characteristics with the highest loading on the five traits most closely related to the human Big Five, and the five characteristics with the highest loading on the chimpanzee-specific factor of Dominance. The characteristics chosen were "inventive", "inquisitive", "unemotional”, "excitable”, “sympathetic", "sensitive", "reckless", “erratic”, “sociable”, “active”, “dominant”, “dependent”, "fearful”, "decisive", and "timid". For each characteristic, the five highest and five lowest-scoring chimpanzees were selected. In the end, we had 30 different chimpanzee images.

\subsubsection{Procedure}

A Latin-square design was used to create five sets of high-low image pairs for each characteristic, such that within a set each high and each low image appeared once (producing five pairs), and across all sets, each low-scoring image for a characteristic was paired with each high-scoring one. The presentation of the high stimulus on the left versus the right of the screen was randomly decided for each trial. For each participant, pairs and characteristics were presented in random order. Participant numbers within each set were balanced as much as possible.

A single trial proceeded as follows. Participants were shown a high/low pair and asked to choose the face best fitting an on-screen definition of a personality characteristic, e.g. 
"more dominant: more able to displace, threaten, or take food from others, or more likely to express high status by decisively intervening in social interactions" (King \& Figueredo, 1997). The image pair and definition were provided onscreen throughout the trial. Responses were made by clicking the chosen face and were not speeded.

After the forced choice trials, participants were shown each chimpanzee photograph used in the prior trials on the computer screen, one at a time, and instructed to rate how old they thought the chimpanzees were on a scale of 1 (young) to 5 (old).

Participants also completed a task involving human faces that will not be included in the present work. The chimpanzee and human trials were blocked separately and the order of the two blocks was counterbalanced across participants. Blocking order had no significant effect on 14 of the 15 characteristic, all $p \mathrm{~s}>.224$, but did affect performance on the characteristic of 'unemotional', $t_{41}=2.34, p=.024$. As indicated below, overall performance on this characteristic was no different from chance, and we did not pursue this effect further.

\subsection{Results and discussion}

Identification accuracy was calculated for each characteristic. All significant findings were related to characteristics loading on human Extraversion. We found accurate performance on the characteristic of 'dominant': accuracy $=0.59 ; t_{42}=3.01, p=.004$, and also 'active': accuracy $=0.72 ; t_{42}=6.76, p<.001$. Interestingly, perception of the characteristic 'sociable' was significantly worse than chance: accuracy $=0.36 ; t_{42}=-4.37, p<$ .0001 , suggesting that information was accurately perceived but systematically misinterpreted. In addition, accuracy on 'sympathetic' (which loads onto human 
Agreeableness) was close to significant: accuracy $=0.56 ; t_{42}=1.84, p=.073$. These results demonstrate that some characteristics relating to Extraversion, and possibly Agreeableness, are accurately perceived in chimpanzee faces.

Participants were also able to accurately estimate chimpanzee age, as age at the time of their rating by zoo keepers was correlated with perceived age as judged by participants, $r_{28}=$ $0.69, p<.001$. We examine effects of age further in Study 2 .

\section{Study 2: accurate identification of dominance in single chimpanzee faces}

This study focused more specifically on characteristics relating to Extraversion and Agreeableness, as motivated by the results of Study 1 . We used a ratings task in order to incorporate more stimuli and generalise our findings beyond one type of methodology. Single chimpanzee faces were presented and rated for different characteristics. Instead of measuring discrimination accuracy, we measured the strength of correlation between real and perceived characteristics.

\subsection{Method}

\subsubsection{Participants}

A different set of 30 students from Bangor University (age 18-27 years, 20 female) took part in exchange for course credit.

\subsubsection{Stimuli}


The same images were used as in Study 1. However, we replaced the image of one chimpanzee with a more extreme personality value for one with a more closed mouth but a less extreme personality value.

\subsubsection{Procedure}

Participants were shown each image on the computer screen, one at a time, and instructed to rate them on a scale from 1 (very low) to 7 (very high). The stimuli were rated on the four characteristics identified in Study 1: sociable, active, dominant, and sympathetic. A description of each characteristic appeared onscreen while that rating was being made. The characteristics were blocked separately, and order of block presentation was counterbalanced between participants. Trials appeared in randomised order for each participant.

Participants also completed a task involving human faces that will not be included in the present work. The chimpanzee and human trials were blocked separately and the order of these two blocks was counterbalanced across participants. We verified that the order of the blocks did not affect performance on any of the chimpanzee judgements, all $p \mathrm{~s}>.05$.

\subsection{Results and discussion}

We calculated the mean rating for each chimpanzee for each characteristic. We then correlated these mean ratings with actual values of chimpanzee characteristics (degrees of freedom therefore reflect the number of chimpanzees judged). Ratings of dominance significantly correlated with actual dominance, $r_{28}=0.42, p=.022$. The interrater reliability of dominance ratings as determined by Cronbach's $\alpha$ was .93 . Pairwise correlations of 
perceived and actual values for other characteristics were not significant, all $p s>.32$. The characteristic of dominance could therefore be accurately assessed, even when participants were unable to directly compare the faces associated with extreme personality values.

Accuracy in dominance ratings did not appear to be affected by age-related cues. Estimates of the ages of individual chimpanzee images (obtained in Study 1) were not correlated with estimates of dominance for those same individual images, $r_{27}=-0.17, p=$ .380. Furthermore, the chimpanzees' actual ages were not correlated with actual dominance, $r_{29}=0.01, p=.964$, meaning that age was in any case not a valid cue for dominance with this group.

We also considered whether dominance ratings might be influenced by implicit sex identification. If male and female chimpanzees differ in dominance, and participants were able to pick up on this cue consciously or otherwise, then accuracy on this task may reflect an implicit ability to identify chimpanzee sex rather than dominance. To see whether this was likely, we compared the actual dominance of males to females in our test set, but these did not significantly differ, $t_{28}=1.48, p=.149$ (although such differences have been reported in other chimpanzee data sets, e.g. Dutton, 2008; King, Weiss \& Sisco, 2008). Likewise the perceived dominance of the two sexes was not significantly different, $t_{28}=0.24, p=.811$. It therefore seems unlikely that people were making their judgements on sex rather than dominance. However, in the next study we tested this possibility directly.

\section{Study 3: within-sex discrimination of dominance}


Here we asked whether people could accurately distinguish levels of dominance within a single-sex group. We presented images of male and female chimpanzees in separate blocks and used forced-choice discrimination to assess accuracy of dominance perception.

\subsection{Method}

\subsubsection{Participants}

A different set of 30 students from Bangor University (age 18-34 years, 22 female) volunteered to participate.

\subsubsection{Stimuli}

From the 30 chimpanzees used in Study 2, three were removed to produce a more controlled set. These included two chimpanzees who had visible facial injuries and one photograph of relatively low image quality. From the remaining 27 chimpanzees (12 females, 15 males), the highest and lowest four male and female chimpanzees for the 'dominance' characteristic were selected. Images were approximately 300 x 300 pixels in size, or about an $8.5 \mathrm{~cm}$ square on the screen.

\subsubsection{Procedure}

Similar to Study 1, high/low pairs of chimpanzee faces were presented onscreen and participants were instructed to select the more dominant one. A definition of 'dominant' was also provided onscreen throughout. Each 'low dominance' face was paired with every 'high 
dominance' face of the same sex, producing 16 male and 16 female pairs. The position of each 'high' face was counterbalanced for side of presentation for each participant. The trials were blocked by stimulus sex, with the order of the pairs randomised within blocks for each participant, and the order of the two blocks counterbalanced between participants.

\subsection{Results and discussion}

Identification accuracy was calculated for each characteristic. Accuracy was significantly above chance when participants were asked to make within-sex comparisons, both for male chimpanzees: accuracy $=0.70 ; t_{29}=6.00, p<.001$, and for female chimpanzees: accuracy $=0.60 ; t_{29}=3.82, p<.001$. In addition, participants were more accurate with male than female chimpanzees, $t_{29}=2.48, p=.019$.

We can therefore be confident that dominance accuracy is present for both male and female chimpanzees. Combined with previous research on human faces (Penton-Voak et al., 2006), we can see that there is some accuracy in characteristics relating to extraversion for both human and chimpanzee faces. Our final study investigated whether accuracy on these two tasks was related.

\section{Study 4: comparing accuracy from human and chimpanzee faces}

Using forced-choice discrimination, we explored accuracy on chimpanzee dominance and human extraversion in order to see whether performances with these two types of stimuli were related. 


\subsection{Method}

\subsubsection{Participants}

A different set of 36 students (age 18-27 years, 26 female) from Bangor University took part in exchange for course credit. Participants completed measures of personality (Donnellan, Oswald, Baird \& Lucas, 2006), empathy (Davis, 1980), and autism (BaronCohen, Wheelwright, Skinner, Martin \& Clubley, 2001).

\subsubsection{Stimuli}

In this study, high/low pairs were created for both chimpanzee and human faces. For the chimpanzees, the set of 27 images from Study 3 were used, and the five chimpanzees scoring highest and lowest on dominance were selected, without regard to sex. For the human discrimination, 36 Caucasian females (see Kramer \& Ward, 2010, for details) were photographed under standard lighting conditions with neutral facial expressions. All jewellery and make-up were removed. Images were cropped to show only the internal facial features, and were converted to black and white (Fig. 1B). These students completed a Big Five personality assessment (Donnellan et al., 2006), and were then sorted for Extraversion, with the highest and lowest five being selected, subject to the constraint that scores on other traits were equivalent. This allowed us to maintain differences between the two sets for Extraversion but minimise other trait differences that may interfere with signal reading. Images were approximately $275 \times 250$ pixels in size, or about $8 \times 7 \mathrm{~cm}$ on the screen.

\subsubsection{Procedure}


Discrimination of human and chimpanzee pairs were made in separate blocks, presented in counterbalanced order between participants. In the chimpanzee blocks, pairs of chimpanzee faces were presented onscreen and participants were instructed to select the more dominant. Each 'low dominance' face was paired with each 'high dominance' face, producing 25 pairs. The position of each 'high' face was counterbalanced for side of presentation for all participants, with each participant seeing each face on one side three times and on the other side twice.

The procedure used with the human faces was identical, although participants were instructed to select the more extraverted. A definition of 'Extraversion' was also provided onscreen throughout: "more talkative, energetic, social, assertive".

\subsection{Results and discussion}

As expected from our previous studies, identification accuracy was again significantly above chance for chimpanzee dominance: accuracy $=0.58 ; t_{35}=3.24, p=.003$. Performance was also accurate on human Extraversion judgements: accuracy $=0.77 ; t_{35}=10.34, p<.001$. However, the within-rater correlation for accuracy on chimpanzee and human faces was not significant, $r_{34}=-0.08, p=.65$. We also saw some individual differences in performance on the tasks. Participant scores on the 'social skills' domain of the Autism Spectrum Quotient were negatively correlated with accuracy on the human discrimination task, $r_{34}=-.37, p=$ .025 , but not the chimpanzee task, $r_{34}=-.01, p=.972$. This negative relationship suggests that those with higher levels of autistic-like traits relating to social skills were specifically worse at reading the signs of extraversion in human faces. 
In addition, chimpanzee accuracy was correlated with participants' conscientiousness scores, $r_{34}=.44, p=.007$. While one could imagine that trying harder on this task may lead to improved accuracy, this does not explain the lack of correlation with human accuracy.

\section{General discussion}

The results of these four studies demonstrate that humans can accurately perceive characteristics relating to extraversion in chimpanzee faces on the basis of static, nonexpressive cues. In particular, people can use cues in both the human and chimpanzee face to identify individuals who are biased towards social activity and dominance-related behaviours.

The ability to detect the characteristic of dominance was an especially robust finding, and it is worth being clear about the nature of this characteristic. The term "dominance" is often used to refer to a situation-specific construct that describes the relationship between individuals and the organisation of the social hierarchy within the group. However, "dominance" as discussed here refers to the broad personality characteristic relating to the chimpanzee's overall competitive tendency or prowess. This characteristic is heritable (Weiss et al., 2000) and reflects a disposition that is relatively stable over time and context. We would expect that these two types of dominance are positively correlated (de Waal, 2000); however, to be clear, it was the personality characteristic, not status per se, that was accurately identified in our studies.

How can we best explain the performance of human observers in accurately decoding the personalities of chimpanzees from their static face images? Clearly there is relevant 
information present in the chimpanzee face, but why would humans be able to accurately process this information? Particularly when, as in our case, these humans have no prior experience of chimpanzees? We hypothesise a shared signal system for personality from the face in humans and chimpanzees, that is, on the basis of their shared evolutionary past, chimpanzees and humans share aspects of a system for communicating behavioural biases to conspecifics

A shared signal system would implicate a variety of physical and psychological systems. These would include (1) shared aspects of personality; (2) shared links between personality and facial morphology; and (3) shared cognitive mechanisms for processing those links. We have already discussed the first point, and the substantive overlap in human and chimpanzee models of personality, as well as the heritability and external validity of chimpanzee personality models to predict behaviour (Pederson et al., 2005; Weiss et al., 2000). Our present results, demonstrating that people are able to identify links between personality and facial morphology in both humans and in chimpanzees, argues strongly for the second point. The point which is not yet demonstrated is the third. Our results show that humans have the cognitive means for processing at least some of the available signals in the face and their associations with personality. However, it is not yet known whether chimpanzees have a similar ability to process and use these signals.

We predict that chimpanzees will be able to identify such signals, at least in the chimpanzee face. Our reasoning is as follows. It is clear that chimpanzees inhabit complex social structures. It is also clear that information in the chimpanzee face, along with associated cognitive abilities, allows chimpanzees to identify important social traits in their conspecifics, such as relatedness and emotional expressions. This information is no doubt 
used to facilitate social interaction. Given these well-agreed facts, and given our present results, demonstrating there are signals relating to extraversion in the chimpanzee face, it would be a surprising evolutionary blindspot if the chimpanzee species were simply unable to process this other type of useful information on their faces. It would be more surprising still that untrained humans were able to use this same information from chimpanzees. We therefore predict that chimpanzees will be able to use the signals that are evidently available from their conspecifics. This evidence, if found, would be a crucial foundation for the more general hypothesis of a shared signal system.

As we reviewed in the introduction, characteristics related to extraversion approximate a universal form of individual difference. These characteristics are widely distributed across mammalian as well as non-mammalian taxa. Extraversion in both humans and chimpanzees is heritable and displays both internal and external validity. The fact that dominance was readily communicated between species suggests the importance of this characteristic in both human and chimpanzee social structures. Indeed, both chimpanzee dominance (King \& Landau, 2003) and human extraversion (Costa \& McCrae, 1980) are correlated with subjective wellbeing. There are of course also important differences in these personality structures, for example as reflected in the six-factor chimpanzee and five-factor human models. These differences may account for some of the errors in cross-species identification.

Our findings also support previous studies measuring signals of trait extraversion from individual human faces (Penton-Voak et al., 2006), that have shown mixed results (Shevlin, Walker, Davies, Banyard \& Lewis, 2003). In addition, while internal features alone have been shown to allow accuracy with composite images (Kramer \& Ward, 2010), our results are the first to demonstrate this with individual photographs, thus demonstrating that the signal for 
extraversion in individual images is still present when information from jawline and skin colour are removed.

From previous studies, it has also been an open question whether accurate personality identification from the human face is based upon the heritable or the acquired components of personality (Kramer \& Ward, 2010; Little \& Perrett, 2007). The fact that humans can identify such signals in chimpanzees clearly indicates some degree of signal which is not specific to any human culture. However, the lack of correlation between accuracy with human and chimpanzee faces in our final study suggests that cultural learning also plays a role. Our participants had little or no experience with the behaviours and faces of any individual chimpanzees, and therefore little room for individual differences in that experience. However, when judging human faces, we would expect people to have a wide range of individual differences in experience which could affect their performance. For example, extraverts demonstrate better visual memory for faces than introverts ( $\mathrm{Li}$, Tian, Fang, $\mathrm{Xu}, \mathrm{Li} \& \mathrm{Liu}$, 2010). Under these assumptions, we might then expect little correlation in performance on human and chimpanzee tasks, for what are essentially statistical reasons.

However, our results still point to some more uniquely human signal in the face. We found in our final study that individual differences in Autism Spectrum Quotient (AQ) measures were correlated with the ability to identify traits in human, but not chimpanzee, faces. As autism is associated with a variety of behavioural deficits in face processing, and specific neurophysiological irregularities (Dawson, Webb, \& McPartland, 2005), an interesting question is whether high-scoring AQ individuals may have social problems relating to difficulties in reading the non-verbal behavioural signals tested in our studies. In any case, while our overall results argue for commonalities between human and chimpanzee 
signal systems, the fact that AQ predicts accuracy on human but not chimpanzee faces also demonstrates some dissociation. This may also be explained in terms of AQ scores affecting the reading of extraversion signals but not those of dominance. However, further research is needed in order to clarify this issue.

Finally, adaptive theories of animal signalling argue that the system is unlikely to be stable unless there is a net advantage to both the sender and the receiver in honest communication (e.g. Krebs \& Dawkins, 1984). It is easy to imagine benefits for the receiver in predicting the personality and subsequent likely behaviours of others. Research has shown that both humans (Jones et al., 2010) and rhesus monkeys (Shepherd, Deaner, \& Platt, 2006) are affected by social status and dominance as reflected in the face during a gaze-cuing task. More interesting is to ask what benefits there may be for the signal sender in this arrangement. While there might be some advantage in concealing likely behaviours and intentions, interpreting our results within a framework of adaptive signalling suggests that in human and chimpanzee social structures, there are also advantages in displaying those behaviours, or at least large costs for concealing them. In conclusion, these four studies provide the first evidence of an honest signal system for personality attributes across species, with evolutionary origins dating back at least to the last common ancestor of chimpanzees and humans. 


\section{References}

Alvergne, A., Huchard, E., Caillaud, D., Charpentier, M. J. E., Setchell, J. M., Ruppli, C. et al. (2009). Human ability to recognize kin visually within primates. International Journal of Primatology, 30(1), 199-210.

Baron-Cohen, S., Wheelwright, S., Skinner, R., Martin, J., \& Clubley, E. (2001). The AutismSpectrum Quotient (AQ): Evidence from asperger syndrome/high-functioning autism, males and females, scientists and mathematicians. Journal of Autism and Developmental Disorders, 31(1), 5-17.

Boothroyd, L. G., Jones, B. C., Burt, D. M., DeBruine, L. M., \& Perrett, D. I. (2008). Facial correlates of sociosexuality. Evolution and Human Behavior, 29(3), 211-218.

Bouchard, T. J., \& Loehlin, J. C. (2001). Genes, evolution, and personality. Behavior Genetics, 31(3), 243-273.

Budaev, S. V. (1997). "Personality" in the guppy (Poecilia reticulata): A correlational study of exploratory behavior and social tendency. Journal of Comparative Psychology, 111, $399-411$.

Carré, J. M., McCormick, C. M., \& Mondloch, C. J. (2009). Facial structure is a reliable cue of aggressive behavior. Psychological Science, 20(10), 1194-1198.

Costa, P. T., Jr., \& McCrae, R. R. (1980). Influence of extraversion and neuroticism on subjective well-being: Happy and unhappy people. Journal of Personality and Social Psychology, 38, 668-678.

Davis, M. H. (1980). A multidimensional approach to individual differences in empathy. JSAS Catalog of Selected Documents in Psychology, 10(4), 85. 
Dawson, G., Webb, S. J., \& McPartland, J. (2005). Understanding the nature of face processing impairment in autism: insights from behavioral and electrophysiological studies. Developmental Neuropsychology, 27(3), 403-424.

de Waal, F. B. M. (2000). Chimpanzee politics: Power and sex among apes (Rev. ed.). Baltimore: Johns Hopkins University Press.

Donnellan, M. B., Oswald, F. L., Baird, B. M., \& Lucas, R. E. (2006). The mini-IPIP scales: tiny-yet-effective measures of the Big Five factors of personality. Psychological Assessment, 18(2), 192-203.

Dutton, D. M. (2008). Subjective assessment of chimpanzee (Pan troglodytes) personality: reliability and stability of trait ratings. Primates, 49(4), 253-259.

Eysenck, H. J., \& Eysenck, M. W. (1985). Personality and individual differences: A natural science approach. New York: Plenum Press.

Freeman, H. D., \& Gosling, S. D. (2010). Personality in nonhuman primates: a review and evaluation of past research. American Journal of Primatology, 71, 1-19.

Goldberg, L. R. (1990). An alternative “description of personality”: The Big-Five factor structure. Journal of Personality and Social Psychology, 59(6), 1216-1229.

Goldberg, L. R. (1993). The structure of phenotypic personality traits. American Psychologist, $48(1), 26-34$.

Gosling, S. D. (1998). Personality dimensions in spotted hyenas (Crocuta crocuta). Journal of Comparative Psycholology, 112, 107-118.

Gosling, S. D., \& John, O. P. (1999). Personality dimensions in nonhuman animals: A crossspecies review. Current Directions in Psychological Science, 8, 69-75.

Jones, B. C., DeBruine, L. M., Main, J. C., Little, A. C., Welling, L. L. M., Feinberg, D. R. et al. (2010). Facial cues of dominance modulate the short-term gaze-cuing effect in human observers. Proceedings of the Royal Society of London B, 277, 617-624. 
King, J. E., \& Figueredo, A. J. (1997). The five-factor model plus dominance in chimpanzee personality. Journal of Research in Personality, 31, 257-271.

King, J. E., \& Landau, V. I. (2003). Can chimpanzee (Pan troglodytes) happiness be estimated by human raters? Journal of Research in Personality, 37, 1-15.

King, J. E., Weiss, A., \& Sisco, M. M. (2008). Aping humans: Age and sex effects in chimpanzee (Pan troglodytes) and human (Homo sapiens) personality. Journal of Comparative Psychology, 122, 418-427.

Kramer, R. S., \& Ward, R. (2010). Internal facial features are signals of personality and health. The Quarterly Journal of Experimental Psychology. Advance online publication.

Krebs, J. R., \& Dawkins, R. (1984). Animal signals: Mind-reading and manipulation. In J. R. Krebs \& N. B. Davies (Eds.), Behavioural ecology: An evolutionary approach (2nd ed., pp. 380-402). Oxford: Blackwell.

Li, J., Tian, M., Fang, H., Xu, M., Li, H., \& Liu, J. (2010). Extraversion predicts individual differences in face recognition. Communicative \& Integrative Biology, 3, 295-298.

Little, A. C., \& Perrett, D. I. (2007). Using composite images to assess accuracy in personality attribution to faces. British Journal of Psychology, 98, 111-126.

Mather, J. A., \& Anderson, R. C. (1993). Personalities of octopuses (Octopus rubescens). Journal of Comparative Psycholology, 107, 336-340.

Maynard-Smith, J., \& Harper, D. (2003). Animal signals. Oxford: Oxford University Press.

Parr, L. A., Hecht, E., Barks, S. K., Preuss, T. M., \& Votaw, J. R. (2009). Face processing in the chimpanzee brain. Current Biology, 19, 50-53.

Parr, L. A., Heintz, M., \& Akamagwuna, U. (2006). Three studies on configural face processing by chimpanzees. Brain and Cognition, 62, 30-42.

Parr, L. A., Waller, B. M., Vick, S. J., \& Bard, K. A. (2007). Classifying chimpanzee facial expressions using muscle action. Emotion, 7(1), 172-181. 
Pederson, A. K., King, J. E., \& Landau, V. I. (2005). Chimpanzee (Pan troglodytes) personality predicts behavior. Journal of Research in Personality, 39(5), 534-549.

Penton-Voak, I. S., Pound, N., Little, A. C., \& Perrett, D. I. (2006). Personality judgments from natural and composite facial images: More evidence for a "kernel of truth" in social perception. Social Cognition, 24(5), 607-640.

Shepherd, S. V., Deaner, R. O., \& Platt, M. L. (2006). Social status gates attention in monkeys. Current Biology, 16, R119-120.

Shevlin, M., Walker, S., Davies, M. N. O., Banyard, P., \& Lewis, C. A. (2003). Can you judge a book by its cover? Evidence of self-stranger agreement on personality at zero acquaintance. Personality and Individual Differences, 35(6), 1373-1383.

Stirrat, M., \& Perrett, D. I. (2010). Valid facial cues to cooperation and trust: male facial width and trustworthiness. Psychological Science, 21(3), 349-354.

Weiss, A., King, J. E., \& Figueredo, A. J. (2000). The heritability of personality factors in chimpanzees (Pan troglodytes). Behavior Genetics, 30(3), 213-221. 
A shared signal system 24

\section{Figure Caption}

\section{Figure 1}

Examples of honest signals of personality. (A) Individual images of two female chimpanzees scoring low (left) and high (right) on dominance, a characteristic that maps onto human Extraversion (70\% of participants accurately identified this specific pair). (B) Examples of stimuli from Study 4: individual face images, with external features removed, of two women scoring low (left) and high (right) on the Extraversion scale (67\% accuracy on this specific pair). 